\title{
Représentation du travail et représentativité syndicale aux marges du salariat : le cas de la distribution directe
}

Representation of work and union representativeness: the case of leaflet distribution

Karel Yon

\section{OpenEdition}

\section{Journals}

Édition électronique

URL : http://journals.openedition.org/travailemploi/5783

DOI : 10.4000/travailemploi.5783

ISSN : 1775-416X

Éditeur

DARES - Ministère du Travail

Édition imprimée

Date de publication : 15 septembre 2012

Pagination : 103-118

ISSN : 0224-4365

\section{Référence électronique}

Karel Yon, «Représentation du travail et représentativité syndicale aux marges du salariat : le cas de la distribution directe », Travail et Emploi [En ligne], 131 | 2012, mis en ligne le 01 décembre 2014 consulté le 30 avril 2019. URL : http://journals.openedition.org/travailemploi/5783 ; DOI : 10.4000/ travailemploi.5783 


\title{
Représentation du travail et représentativité syndicale aux marges du salariat : le cas de la distribution directe
}

\author{
Karel Yon $\left.{ }^{*}\right)$
}

La réforme de la représentativité syndicale permet-elle de lutter contre l'institutionnalisation des syndicats? L'article s'interroge sur cette attente, qui se retrouve autant chez les acteurs de la réforme que chez les spécialistes du syndicalisme. En étudiant un secteur professionnel de constitution récente, la distribution directe, il montre que la réforme ne saurait répondre à un problème incorrectement formulé. L'institutionnalisation des syndicats est en effet un processus contradictoire, qui dissocie autant qu'il relie les salariés à leurs représentants. Cette précision conduit à étudier l'impact de la réforme de la représentativité syndicale de manière plus nuancée. La mobilisation des règles de représentativité dans les deux entreprises étudiées montre que les effets de la réforme en termes de syndicalisation s'avèrent ambivalents. Le nouveau régime de représentativité ne garantit pas davantage un contrôle plus étroit des salariés sur leurs représentants. Il accroît en revanche l'interdépendance des arènes de branche et d'entreprise, en tant que celle-là est désormais connectée à celles-ci via la procédure élective.

C'est un lieu commun : les syndicats seraient trop institutionnalisés, ce qui les conduirait à agir en vase clos, à négocier au nom de tous sans rendre de compte à personne. C'est pour répondre à ce problème qu'a été adoptée la loi du 20 août 2008 «portant rénovation de la démocratie sociale et réforme du temps de travail». Ainsi, peu de temps avant la signature de la position commune dont la loi allait reprendre l'essentiel, le secrétaire général de la Confédération française démocratique du travail (CFDT) François Chérèque interprétait cette négociation comme un moyen de rapprocher les organisations syndicales des salariés : «Le syndicaliste est présent dans l'entreprise, le salarié le connaît. Les organisations syndicales, elles, sont vues comme des institutions appartenant à l'élite décisionnelle. Je pense, moi aussi, que les syndicats sont trop institutionnalisés. C'est pour cela que la représentativité des syndicats doit être définie au sein des entreprises» ${ }^{(1)}$. Au même moment, on retrouvait une argumentation similaire du côté de la Confédération générale du travail (CGT), qui reprochait au décret de 1966 de donner à voir les syndicats «comme des institutions immuables, séparées de l'expression des salariés qu'ils représentent» et appelait à ce que la représentativité « découle du vote des salariés dans les élections professionnelles [à] tous les

(*) CERAPS (UMR 8026) - Université Lille 2; karel.yon@ univ-lille2.fr

(1) «Le dialogue social sera toujours un combat», entretien avec François Chérèque, Challenges, $\mathrm{n}^{\circ}$ 117, 27 mars 2008, pp. 66-68. niveaux (entreprise, branche, bassin d'emploi, région, nation)» (2). En abrogeant la présomption irréfragable de représentativité des cinq confédérations «historiques» au profit d'une procédure fondant celle-ci sur le vote, la réforme de 2008 vise à constituer des organisations professionnelles «fortes et légitimes» ${ }^{(3)}$. Non seulement elle inciterait les syndicats à tenir compte du vote des salariés dans leur pratique de négociation (en interdisant notamment les accords minoritaires) mais elle jouerait même un rôle d'incitation à la syndicalisation, en contraignant les organisations syndicales à disposer d'une base militante suffisamment solide et étendue pour faire face aux épreuves électorales. $\mathrm{Au}$ regard des faiblesses syndicales dans d'importants secteurs du salariat - que l'on songe aux TPE (très petites entreprises) et PME (petites et moyennes entreprises), au secteur des services, aux jeunes et aux travailleurs précaires -, la réforme de la représentativité syndicale a été défendue par ses promoteurs comme un début de solution à la crise de la représentation syndicale, crise que les seuls efforts volontaristes des organisations n'ont jusqu'alors pas été capables

(2) «Négociation sur la réforme de la représentativité. État des lieux et propositions de la CGT. Fiche 3 . Une représentativité des organisations syndicales administrée», consulté le 28 avril 2008 sur le site de la CGT:

$\mathrm{http}: / / \mathrm{www}-\mathrm{v} 2 . \mathrm{cgt} . \mathrm{fr} /$ internet $/ \mathrm{html} / \mathrm{lire} /$ ?id doc $=5836$

(3) Dossier législatif relatif à la loi $\mathrm{n}^{\circ} 2008-789$ du 20 août 2008 portant rénovation de la démocratie sociale et réforme du temps de travail - Exposé des motifs. Site Légifrance, consulté en ligne le 5 juin 2009:

http://www.legifrance.gouv.fr/affichLoiPubliee.do;jsessionid= D99E2D863E86CC701892AF7F0435223E.tpdjo14v_1?idDoc ument=JORFDOLE000019017524\&type $=$ expose 
d'enrayer ${ }^{(4)}$. Mettre cette assertion à l'épreuve supposerait d'investir les multiples terrains correspondant aux différents champs de syndicalisation possibles. Cet article se propose d'apporter une première contribution à ce travail de documentation à partir d'un secteur d'activité. La distribution d'imprimés non adressés (prospectus, journaux gratuits d'annonces, publications institutionnelles) dans les boîtes à lettres, que les acteurs du secteur appellent «distribution directe», est un cas intéressant à plusieurs titres. Il s'agit d'abord d'un domaine emblématique de la «nouvelle frontière» syndicale. L'émergence de ce secteur est illustrative de l'essor des services marchands dans notre économie, où les syndicats sont moins bien implantés que dans l'industrie ou dans les fonctions publiques. Son autonomisation, très récente, témoigne de la recomposition des frontières entre secteurs public et privé. $\mathrm{La}$ forme d'emploi dominante est celle du travail à temps partiel, statut atypique qui génère une forte précarité (voir encadré 1).

\section{Encadré 1 \\ La distribution directe : une précarité institutionnalisée?}

Si ses origines sont plus anciennes, l'activité de distribution d'imprimés non adressés s'est développée dans les années 1970, parallèlement à l'essor de la presse gratuite d'annonces et de la grande distribution. Avec le déclin de la presse d'annonces, illustré par la récente liquidation de Paru/Vendu, et l'essor des sites d'annonces sur internet (comme Le Bon coin), les entreprises de distribution ont investi le domaine de la communication virtuelle tandis que le travail des distributeurs se recentrait sur la diffusion de prospectus publicitaires, de publications institutionnelles éditées par les collectivités territoriales et, depuis peu, de la nouvelle presse d'information gratuite (type Métro ou 20 Minutes).

Bien que l'activité soit extrêmement éclatée, elle est désormais contrôlée pour l'essentiel par Gratuit, filiale du groupe La Poste, et Distrib, filiale d'un important groupe de presse, qui se partagent $95 \%$ du marché. Les deux entreprises sont de taille équivalente. Les effectifs de la première sont moins importants mais son chiffre d'affaires est supérieur (430 millions d'euros, contre 312,9 millions pour Distrib). D'après l'Association pour le financement des activités du spectacle (AFDAS), qui gère les contributions formation des entreprises de la distribution directe, il existait cependant, en 2010, 43 sociétés de distribution qui cotisaient à cet organisme professionnel collecteur agréé. La plupart sont des micro-sociétés qui échappent souvent aux règles de la branche; quelques entreprises de plus de 50 salariés interviennent en régions (parfois en sous-traitance de commandes passées à l'un ou l'autre "grand").

Les salaires sont faibles et les conditions de travail difficiles, donnant régulièrement lieu à des articles «édifiants » dans la presse locale ou nationale. Les données présentées dans le bilan social de branche permettent de se faire une idée de la condition des distributeurs. Ces données portent sur les deux principales entreprises du secteur, Gratuit et Distrib, qui restent seules adhérentes au Syndicat professionnel de la distribution directe. En 2011, il y avait 32985 distributeurs, dont $40 \%$ de femmes, pour 3269 salariés «permanents" chargés de les encadrer. L'effectif total de la branche, selon l'AFDAS, est de 22500 salariés en équivalent temps-plein, ce qui souligne l'importance du travail à temps partiel. La main-d'œuvre est plutôt âgée : la moitié des distributeurs a plus de 50 ans, un huitième d'entre eux a moins de 30 ans. La quasi-totalité des distributeurs est en CDI, même si un quart des embauches s'effectue en CDD. Le turn-over est extrêmement important, avec presque autant de départs que d'embauches (32750 pour 33279 ), dont un quart de départs par démission et près de la moitié en fin de période d'essai, ce qui témoigne de la difficulté des entreprises à stabiliser la force de travail. Seuls $5 \%$ des distributeurs sont employés à temps plein, les deux tiers sont embauchés sur des contrats à temps partiel inférieurs à 65 heures mensuelles, le plus souvent en-dessous du plafond d'ouverture des droits à la sécurité sociale (60 heures mensuelles). Le salaire moyen indiqué en 2011 était de $628 €$ pour les femmes et $655 €$ pour les hommes. Dans la mesure où la distribution est avant tout une industrie de main-d'œuvre, fortement dépendante des clients «grands comptes " que sont les grandes surfaces, la rémunération des distributeurs reste la principale variable d'ajustement économique. En pratique, les distributeurs travaillent bien souvent pour des durées excédant le nombre d'heures pour lesquelles ils sont payés, ainsi que l'attestent régulièrement des condamnations pour "travail dissimulé".

La flexibilité des horaires fait de la distribution directe une activité aussi bien prisée par les jeunes, les femmes ayant des charges de famille que les travailleurs ayant besoin de cumuler plusieurs salaires. L'absence de qualification requise ainsi que la facilité d'embauche attirent également beaucoup de travailleurs immigrés, des retraités cherchant un complément de revenu (militaires et policiers en retraite, pensionnés paupérisés) ou des individus ayant connu des accidents biographiques (commerçants faillis, salariés en rupture de ban). II n'existe pas de réels collectifs de travail : les distributeurs sont dispersés sur plusieurs centaines de sites, répartis sur tout le territoire, sur lesquels ils sont rarement présents. Ils viennent y chercher chaque semaine leur lot d'imprimés à distribuer et effectuent seuls leur tournée.

NB : Bien que la structure quasi-duopolistique de la branche rende l'opération artificielle, le nom des entreprises a été modifié.

(4) Réorganisation des structures, objectifs chiffrés d'accroissement des effectifs adhérents, ciblage des fractions sous-représentées du salariat, renouvellement et diversité des responsables syndicaux, etc. Ces enjeux ont été formulés dès le milieu des années 1980 à la CFDT (Guillaume, Pochic, 2009) et au début des années 2000 à la CGT (Piotet, 2009).

- $104 \cdot$ Travail et Emploi $n^{\circ} 131$ 
La distribution directe cumule donc un ensemble de traits qui sont autant de défis pour un «renouveau syndical»(5). Loin des expérimentations menées en France ou à l'étranger pour organiser les travailleurs précaires (BÉroud, Bouffartigue, 2009), l'intervention syndicale a cependant surtout relevé dans ce secteur d'un registre d'action traditionnel, celui de la négociation collective. La structuration de la branche professionnelle résulte d'une convention collective nationale $(\mathrm{CCN})$ adoptée récemment, au milieu des années 2000, après dix années de négociation. La reconnaissance du fait syndical s'est d'emblée jouée sur le terrain institutionnel et elle n'a pas semblé mettre fin, ni à la précarité du secteur, ni à la conflictualité sociale qui reste endémique. On retrouve ainsi une situation proche de celle décrite par JeanMichel Denis (2008) dans le secteur du nettoyage industriel : il y pointe le problème de la précarité des salariés et leur dissémination sur de multiples sites qui empêche les syndicats de «disposer d'un maillage territorial dense» pour faire respecter les normes conventionnelles sur les chantiers (p. 48). Il mentionne également le «problème classique de la "distance" ou de l'éloignement entre syndicats et salariés, générés par le mécanisme de la représentation, la bureaucratisation des organisations et/ ou l'institutionnalisation du syndicalisme» (p. 45). La distribution directe offrirait-elle une illustration analogue de cette déconnexion entre les travailleurs et leurs représentants? Dans ce cas, la loi du 20 août 2008 «portant rénovation de la démocratie sociale et réforme du temps de travail» aurait-elle permis d'y remédier? Sans prétendre à aucune possibilité de généralisation quant à ses résultats empiriques, ce texte n'entend pas moins apporter des éléments de réflexion qui dépassent le cadre monographique en éclairant d'un jour nouveau la question de l'institutionnalisation des syndicats.

Dans la plupart des discours sur le syndicalisme - qu'ils soient tenus par des chercheurs, des journalistes, les acteurs politiques et administratifs ou les syndicalistes eux-mêmes - l'institutionnalisation est prise dans un sens négatif : elle désigne le processus d'autonomisation, de clôture et de bureaucratisation des organisations qui expliquerait, au moins en partie, la crise du mouvement syndical (6). Dominique Andolfatto et Dominique Labbé insistent depuis de longues années sur la «faiblesse des racines sociales du syndicalisme». Pointant une «représentativité de plus en plus problématique», ils dépeignent les syndicalistes comme des «professionnels de la représentation [...] qui n'ont plus de liens, autres qu'électoraux, avec les salariés qu'ils

(5) Pour une synthèse en français sur cette littérature, voir Thomas, 2011

(6) On est de ce point de vue très loin des conceptualisations développées autour du champ dynamique et pluriel des sociologies de l'institutionnalisation (LAgroye, OfFerlé, 2010; TOURNAY, 2011). sont censés représenter et qui n'ont plus qu'une connaissance assez théorique de la situation réelle et des attentes de ces salariés 》 (ANDOLFATto, LaBbÉ, 2006, p. 349; p. 351). Cette analyse perpétue une problématique ancienne : la critique de l'institutionnalisation des organisations syndicales, née à l'aube du mouvement ouvrier avec la «loi d'airain de l'oligarchie» (Michels, 1971), a connu en France un important regain dans les années 1980, à l'occasion d'un improbable chassé-croisé entre l'effondrement des effectifs syndicaux et l'accroissement de la puissance institutionnelle des syndicats (ADAM, 1983). Elle a été plus précisément formulée par Pierre Rosanvallon, qui explique l'institutionnalisation du syndicalisme par l'autonomisation de sa fonction d' «agence sociale», à savoir «l'ensemble des fonctions syndicales qui sont liées à un rôle institutionnel [...]. En tant qu'agence sociale, le syndicat est une sorte de "fonctionnaire du social", un organisme quasi public. Cet aspect se distingue de sa dimension de mouvement social (force de revendication et de négociation)» (RosanVAllon, 1998, p. 24). La réforme de la représentativité syndicale, dans ses justifications comme dans ses critiques, s'appuie généralement sur ce diagnostic. Pour certains, elle serait un moyen d'ajuster la représentation syndicale au monde du travail réel en reconnectant, par la voie des urnes, les salariés à leurs représentants (BÉVORT, JOBERT, 2008). D'autres ont pronostiqué un effet inverse à celui attendu : celui d'aggraver l'institutionnalisation des syndicats et, ce faisant, leur déracinement social (Andolfatto, LabBÉ, 2009). Ces points de vue opposés partagent ainsi le constat d'un décalage entre le monde du travail «réel » et sa représentation syndicale. C'est ce présupposé que je voudrais discuter dans cet article.

À mon sens, l'institutionnalisation ne peut pas seulement être pensée comme un processus de différenciation éloignant la représentation syndicale de sa «base» sociale. Je voudrais montrer qu'il est nécessaire de décrire cette dynamique dans sa nature contradictoire, comme un mouvement ayant aussi pour effet de lier plus étroitement les syndicats aux travailleurs. Cette démarche est sous-tendue par une approche «constructiviste» du salariat. Elle pose, à l'encontre de l'hypothèse de disjonction entre le monde du travail «réel» et sa représentation syndicale, que l'un et l'autre participent des institutions du salariat (VAtin, Bernard, 2007; Friot, 2012). En tant que «dispositifs de connaissance et d'action», les institutions organisent les activités économiques (SALAIS, 2008) autant qu'elles façonnent les «travailleurs» dans leur être social comme dans leur subjectivité (CASTEL, 1995). Et le syndicalisme participe doublement de cette structuration, comme forme instituée et force instituante : si «le droit du travail s'est largement construit en construisant les syndicats » (BORENFreund, Souriac, 2008, p. 2), c'est parce que les syndicats, en retour, se sont construits en construisant le droit du travail. Dans cette 
perspective, l'institutionnalisation des syndicats doit être comprise dans le cadre plus large d'une formalisation juridique croissante des relations de travail, la négociation de branche étant un vecteur-clé de ce maillage juridique (DiDRY, 2002). Une telle grille d'analyse, qui s'appuie sur une enquête de terrain approfondie (voir encadré 2), implique de rompre avec une conception mécaniste et homogénéisante du droit pour le considérer dans sa dualité et sa plasticité, à la fois «cadre idéologique structurant les relations de travail, et ressource matérielle et symbolique dont l'usage peut changer la teneur et les formes de domination qui y ont cours» (PÉLISSE, 2009, p. 82).

\section{Encadré 2 \\ Matériaux et sources}

Cet article s'appuie sur des matériaux recueillis dans le cadre d'une recherche en cours sur l'application de la réforme d'août 2008 «portant rénovation de la démocratie sociale»(1). J'ai interviewé, depuis mars 2010, divers acteurs du secteur : deux représentants de l'administration du Travail, seize négociateurs et représentants syndicaux de branche, délégués et militants syndicaux des deux principales entreprises de distribution directe, le représentant d'un syndicat «libre" issu de l'encadrement et un représentant d'une direction d'entreprise. J'ai en outre pu consulter les dossiers du représentant de la Direction générale du travail (DGT) ayant suivi, plus ou moins directement, les relations professionnelles dans le secteur depuis 2000. On y trouve des comptes rendus de commissions mixtes paritaires, des courriers des partenaires sociaux à la DGT, des courriers internes à la DGT, divers documents syndicaux, et les "fiches de liaison», comptes rendus d'activité réalisés par les "Présidents de commission mixte» (PCM) qui représentent le ministère du Travail lors des séance de négociation collective. J'ai amassé de nombreux matériaux (tracts syndicaux, témoignages et analyses, articles de presse) sous forme papier ou sur les sites internet des syndicats et sur plusieurs blogs et forums de salariés du secteur. Enfin, j'ai assisté à trois jours de réunion/formation de militants et délégués syndicaux issus des deux entreprises, ainsi qu'à la réunion d'une commission mixte et d'un groupe de travail paritaire au niveau de la branche.

(1) Recherche pour la Dares (2010-2012) menée avec Sophie Béroud, Jean-Michel Denis, Marnix Dressen, Maïlys Gantois, Cécile Guillaume et Donna Kesselman. La distribution directe (DD) n'est qu'un secteur parmi d'autres couverts dans le cadre de cette enquête.

Dans un premier temps, j'évoquerai l'histoire relativement récente de la branche, qui se caractérise par l'institution conjointe du secteur et des organisations syndicales appelées à agir en son sein. Je montrerai les limites d'une lecture en termes de déconnexion entre les salariés et leurs représentants.
Décrire les conditions d'institutionnalisation de la branche de la distribution directe permet, en retour, de mettre en perspective les implications de la loi de 2008. On ne peut en effet saisir les changements induits par le nouveau régime de représentativité qu'en comparant la séquence ouverte par cette réforme aux séquences antérieures. Dans une deuxième partie, je montrerai ainsi de quelle manière la mise en œuvre des nouvelles règles de représentativité transforme la réalité syndicale dans le secteur, tant au plan de la représentation des salariés que de la pratique du dialogue social. À cet égard, la configuration du secteur, assez singulière puisqu'il s'agit d'un quasi-duopole, facilite la comparaison et l'analyse des interactions entre le niveau de la branche professionnelle et celui des entreprises.

\section{Institutionnalisation et syndicalisation de la distribution directe}

Selon le schéma classique de l'institutionnalisation, que l'on retrouve aussi bien chez RosanVaLLon et ses continuateurs (Duclos, Mériaux, 2009) que dans la littérature néo-corporatiste (par exemple : OFFE, 1985), le syndicat qui voit son pouvoir garanti par l'État ne se préoccupe plus de son implantation réelle dans le salariat. La réalité vivante du travail lui échappe progressivement au risque de perdre sa capacité d'intermédiation. Ce schéma est a priori bien adapté pour faire le récit de l'implantation syndicale dans le secteur de la distribution directe, qui semble s'opérer essentiellement «par le haut». Deux séquences peuvent être distinguées : l'institution, qui correspond à la phase d'élaboration des normes conventionnelles, et l'institutionnalisation, qui correspond à la phase ultérieure de routinisation des règles.

\section{Institution de la branche et reconnaissance du fait syndical}

L'activité de distribution d'imprimés non adressés fut longtemps dispersée entre des univers professionnels très différents : d'une part le monde postal, d'autre part la presse et la publicité. La première tentative d'introduction d'imprimés publicitaires dans les tournées des facteurs, au cours des années 1953-56, avorta en raison de la résistance syndicale. Elle fut réitérée au début de la décennie 1970 sur un mode volontariste, contribuant à diviser les agents selon qu'ils acceptaient ou non cette tâche qui était assortie d'une rémunération complémentaire (CARTIER, 2003). Dans les univers professionnels du secteur privé, régies publicitaires et sociétés de presse, l'activité était, à la manière du portage de presse, principalement payée à la tâche. Le tâcheronnat est un contrat de prestation de service qui relève du droit des affaires : l'«entreprise achète une prestation de service globale décomptée en 
nombre de pièces à réaliser et dont elle a négocié le prix unitaire, ce qu'on appelle dans le jargon un “chantier" ou un "lot"» (CÉLÉRIER, 2012, p. 91). Ici, le chantier se définit en nombre de boîtes aux lettres à distribuer. Pour les entreprises qui recourent à ce type de prestation, c'est un moyen d'externaliser les coûts autant que les risques du travail. Les travailleurs, appelés distributeurs, effectuent une partie du travail à domicile (la préparation des «poignées», c'est-à-dire des liasses de prospectus qui seront glissés dans les boîtes à lettres) et fournissent leurs propres outils de travail (chaussures de marche et vêtements pour faire face aux intempéries, véhicule pour se rendre sur le lieu du chantier, chariot pour transporter les poignées, etc.). Ils sont seuls responsables des conditions de réalisation de la prestation, ce qui se traduit notamment par la mobilisation de la famille, conjoint-e et enfants apportant gratuitement leur contribution à la tâche. Dénigrée par les syndicats de facteurs attachés à l'esprit de service public qui la voyaient comme un «sale boulot» commercial, l'activité était peu visible dans le privé. Donnant lieu à de multiples arrangements informels, négociés de gré à gré entre les distributeurs et les donneurs d'ordre, elle était d'autant plus considérée comme périphérique par les syndicats de la presse ou de la publicité qu'elle était difficilement saisissable.

Rémunérés à la tâche, les distributeurs se voyaient délivrer des contrats qui n'offraient aucune garantie de respect du SMIC (salaire minimum interprofessionnel de croissance) horaire, ce qui était illégal. C'est pourquoi la Direction générale du travail, au début des années 1990, invite les entreprises intervenant dans ce domaine d'activité à s'entendre avec les confédérations présumées représentatives pour donner aux travailleurs une couverture conventionnelle, dans la continuité du projet de généralisation des conventions de branche lancé à la fin des années 1970 (TALLARD, 2004). Une responsable du syndicat FO (Force ouvrière) de l'édition, de la presse et de la publicité, qui fut parmi les artisans de la convention de branche, souligne le caractère réactif de ce «concernement» syndical : «On s'est plongé dans le dossier, qu'est-ce que la distribution directe? On $n$ 'avait pas de syndicalisation évidemment» ${ }^{(7)}$. Les syndicats sont invités à formuler des propositions : élargissement du domaine d'application d'une convention existante ou négociation d'un texte $a d$ hoc. La position de cette syndicaliste est de négocier une annexe à la convention collective de la logistique de publication directe. Mais la création d'une organisation patronale spécifique, le Syndicat de la distribution directe (SDD), en 1994, réoriente la négociation vers la production d'un texte autonome. Au départ, la convergence d'intérêts est manifeste entre le patronat et les syndicats. La négociation au niveau national est, pour les syndicats, un moyen

(7) Entretien, 24 janvier 2012. de compenser une présence défaillante sur le terrain des entreprises. Une telle pratique de la négociation de branche est connue. Elle contribua notamment beaucoup à l'inscription de Force ouvrière dans le paysage syndical (BERGOUNIOUX, 1975). De son côté, contraint par la puissance publique à «entrer dans le droit», le SDD est prêt à tolérer les organisations syndicales dans l'arène de branche tant qu'elles restent hors des entreprises ${ }^{(8)}$. Doit-on pour autant parler de syndicalisation par le haut, avec pour conséquence une tare originelle d'organisations «hors-sol», confinées dans l'arène paritaire, davantage portées par la reconnaissance institutionnelle que par les distributeurs? En réalité, la dynamique de la négociation change progressivement la donne.

Dans la phase initiale d'élaboration des règles conventionnelles, la négociation collective de branche contribue à stimuler l'implantation syndicale. Si les premiers négociateurs syndicaux sont extérieurs au secteur (ce sont des permanents syndicaux fédéraux issus du monde de la presse ou de la publicité), ils partagent le souci de se lier aux distributeurs. Les organisations syndicales s'implantent dans les entreprises en y relayant l'ambition, proclamée sur la scène paritaire, de «faire de la distribution un vrai métier» et en contestant le paiement à la tâche devant les tribunaux et les conseils de prud'hommes ${ }^{(9)}$, ou lors de grèves sur les lieux de travail. La négociation de branche est ainsi rythmée et conditionnée par les échanges de coups qui se jouent sur d'autres scènes. De même, la participation de travailleurs de la branche aux délégations syndicales est un enjeu d'affrontement avec le SDD, à qui les syndicats reprochent par moments de ne pas tenir ses engagements en matière de prise en charge des frais de déplacement, condition pour que des non parisiens (et non permanents) participent aux négociations. La présence de travailleurs de la distribution apporte un savoir d'expérience qui nourrit les échanges, mais comme l'attestent les comptes rendus de réunions, ce sont les permanents fédéraux qui mènent les négociations. En plus de mobiliser des compétences variées, notamment rhétoriques et juridiques, et de coordonner l'intervention syndicale sur ces multiples scènes, les permanents assurent une continuité quand les travailleurs issus du rang changent régulièrement : «Nous, nos délégués ont toujours été licenciés », me dit un responsable

(8) Plus précisément, il existait une présence syndicale dans certaines entreprises, mais elle était tolérée dans la mesure où elle acceptait la logique du travail à la tâche, comme en témoignent quelques accords d'entreprise négociés au début des années 1990; sinon, la répression était de mise.

(9) Des recours devant les prud'hommes conduisent régulièrement à la requalification des contrats de distributeurs en CDI à temps plein. Le problème du contrat de travail des distributeurs est pointé de manière récurrente par les inspecteurs du travail ou devant les juridictions, comme les tribunaux d'instance, à partir de l'enjeu connexe de la représentation du personnel (celle-ci se détermine en effet à partir des effectifs calculés en équivalents temps plein). 
cégétiste interviewé(10). Il entend ainsi attester de la probité de sa délégation, reprochant à certains syndicats d'avoir enrôlé dans la leur des salariés dont l'indépendance vis-à-vis de l'employeur était moins évidente. Mais il témoigne également de la fragilité de l'implantation syndicale, confirmant les propos de la permanente FO interviewée selon laquelle, avant l'institution de la $\mathrm{CCN}$, «la représentation syndicale était ridicule et éphémère».

La négociation se dénoue avec l'arrivée dans le camp patronal d'un acteur mieux disposé vis-à-vis $\mathrm{du}$ «dialogue social». La fusion-acquisition d'un opérateur historique du marché avec la société Gratuit(11), propriété du groupe La Poste, signe en 2003 l'entrée de l'opérateur public sur le marché de la distribution privée. Avec lui, les syndicats de la Poste pénètrent le secteur, ils organisent à leur tour des distributeurs, parfois même en concurrence avec des syndicats ayant la même affiliation confédérale(12), et investissent la négociation. Gratuit proclame vouloir diffuser le «modèle social» du groupe auprès des distributeurs et encourage un certain nombre de concessions. La société généralise les CDI à temps partiel, prend des engagements en termes d'amélioration des conditions de travail, de formation professionnelle, etc. Ces accords noués dans l'entreprise précèdent et poussent à la conclusion de l'accord de branche.

Ainsi, un compromis se scelle in fine dans la Convention collective nationale $(\mathrm{CCN})$ de la distribution directe. Le texte est signé le 9 février 2004 par le SDD et des syndicats et fédérations affiliés aux cinq confédérations. Il est étendu par arrêté du ministère du Travail le 16 juillet 2004 et entre en vigueur l'année suivante, le 1 er juillet 2005 . Le principal changement induit par l'institution de la CCN consiste dans l'abandon officiel du travail à la tâche. La rémunération de l'activité de distribution est depuis lors basée sur une mesure temporelle, consignée dans des contrats de travail à durée indéterminée. Mais le statut de distributeur, qu'organise la $\mathrm{CCN}$, n'en reste pas moins atypique : il combine le contrat de travail à temps partiel modulé(13) à un système de rémunération particulier, appelé «préquantification», qui évalue a priori le temps nécessaire à la réalisation des chantiers de distri-

(10) Entretien, 24 janvier 2012.

(11) Gratuit a été créée en 1987 comme filiale de la Poste proposant des services de marketing direct aux entreprises. Elle étend son activité à la distribution de publicité non adressée en fusionnant avec une société de diffusion rachetée à un groupe de presse concurrent du groupe propriétaire de Distrib.

(12) C'est le cas en particulier des fédérations CGT de la Poste et du Livre.

(13) La modulation désigne la possibilité créée par un accord collectif de faire varier la durée hebdomadaire du travail sans recourir aux heures supplémentaires si, en moyenne annuelle, la durée hebdomadaire n'excède pas la durée mentionnée au contrat de travail. Ce dispositif a été adopté dans plus de la moitié des accords RTT (réduction du temps de travail) entre 1998 et 2000 (PÉLISSE, 2003). bution. Ce système répond au souci patronal de «flexibilité» face aux demandes du marché en dérogeant au droit commun du travail qui impose un décompte ex post des heures de travail effectuées. Le compromis de la CCN, avalisé par la Direction générale du travail, échange ce statut dérogatoire contre une forte reconnaissance des syndicats, à travers un droit syndical important qui concerne autant la branche que les entreprises (voir encadré 3).

\section{Encadré 3}

\section{Le droit syndical dans la Convention collective nationale de la distribution directe}

La CCN prévoit des dispositions communes à tout le secteur en matière de droit syndical et de représentation du personnel. Trois d'entre elles méritent d'être soulignées. La première concerne les entreprises : étant donné la dispersion des centres de distribution et la fréquence des «petits contrats" à temps partiel, la CCN prévoit qu'un coefficient multiplicateur de 1,7 soit appliqué au calcul des effectifs en équivalents temps plein, calcul qui permet de délimiter les établissements, de déterminer le nombre de mandats dans les institutions représentatives du personnel (IRP) et le volume d'heures de délégation. La seconde disposition est relative aux heures de délégation : elles sont payées comme temps de travail effectif quand elles sont prises pendant les horaires de travail du représentant du personnel (RP); prises en dehors des heures de travail, elles sont considérées comme des heures complémentaires ou supplémentaires, calculées sur la base du SMIC. La troisième disposition porte sur la branche : la CCN prévoit que chaque organisation syndicale signataire dispose d'un "conseiller technique", chargé du suivi des affaires de la branche, en la personne d'un salarié à temps plein d'une des entreprises du secteur, détaché dans cette fonction. II s'agit d'une disposition assez exceptionnelle : il est peu fréquent qu'un syndicat patronal rémunère directement l'action syndicale.

Le processus de syndicalisation a bien été impulsé «par le haut», mais il ne se réduit pas à un syndicalisme de sommet : la reconnaissance du fait syndical dépasse le cadre de l'arène paritaire pour s'imposer dans les entreprises, dans le cours même de la négociation et ensuite dans le texte de la CCN.

\section{Malaise dans la représentation : la difficile légitimation du compromis de branche}

Si l'hypothèse d'une «syndicalisation de sommet » est erronée, serait-il plus approprié d'opposer la phase initiale d'institution, fondatrice, propice à la syndicalisation car requérant la mobilisation active des intérêts, et la phase ultérieure d'institutionnalisation, marquée par la démobilisation des 
distributeurs et l'autonomisation d'un intérêt propre à leurs représentants? On retrouverait ainsi le schéma selon lequel la représentativité légale étant acquise, l'activation de la représentativité sociale deviendrait inutile. À la phase initiale de communion succèderait la phase d'assoupissement ou de différenciation des intérêts, ayant pour conséquence un «risque de délégitimation pour des organisations qui, assurées de se perpétuer, n'ont plus d'intérêt ou de capacité à démontrer leur représentativité sociale» (OfFerlé, 1998, p. 72). On constate en effet diverses manifestations de défiance à l'égard des organisations associées au compromis de branche, ce qui a permis à de nouveaux acteurs prétendant à la représentation des distributeurs d'émerger. Pour autant, ces phénomènes ne sauraient être résumés au schéma d'une déconnexion entre des syndicats institutionnalisés et des distributeurs mécontents.

La contestation a d'abord surgi du sein des syndicats eux-mêmes, au moment de la signature de la convention collective. L'acceptation d'un compromis dérogatoire n'allait pas de soi pour certains syndicalistes dont l'action sur le terrain s'était faite au nom de la normalisation salariale du secteur. Un interviewé évoque ainsi le cas d'un distributeur représentant la CFTC (Confédération française des travailleurs chrétiens) «qui venait aux commissions avec son code du travail sous le bras» et qui fut écarté par sa confédération qui lui reprochait de bloquer la conclusion de la négociation. Certains syndicats de la Poste ont été les plus critiques par rapport à l'issue de la négociation, voyant dans la formalisation du statut de distributeur un risque, à terme, pour le statut de facteur. Ainsi, la FAPT-CGT a refusé de signer la CCN, mais a été dissuadée de manifester son opposition à l'issue d'arbitrages internes à la confédération, la signature de la CGT étant apportée par la FILPAC (14). Mais les tensions ont surtout eu lieu entre les acteurs investis sur la scène paritaire et les militants présents dans les entreprises, comme en témoigne un représentant syndical CFDT :

«[la $\mathrm{CCN}]$ elle a été mise en place à Gratuit le 5 juillet [2005], on a fait grève le 5 juillet. [...] Je vous cacherai pas qu'en tant que délégué central, en 2005 j'ai envoyé un recommandé à ma fédération en disant il faut dénoncer cette convention. » ${ }^{(15)}$

Dans leur traduction concrète au sein des entreprises, les nouvelles règles issues du compromis de branche se traduisent en effet par une baisse de la rémunération directe. Un distributeur entré à Gratuit en 2000, ancien militant CGT, s'est prononcé avec d'autres contre la CCN : «on avait fait la comparaison des fiches de paye». Un autre, employé de Gratuit bien avant le rachat par le groupe La Poste, ancien militant de l'Union nationale des syndicats

(14) La FAPT est la Fédération des activités postales et des télécommunications; la FILPAC, la Fédération des travailleurs des industries du livre, du papier et de la communication.

(15) Entretien du 12 avril 2012. autonomes (Unsa), note qu'avec l'entrée en vigueur de la convention, son salaire a été divisé par trois. C'est ce que résume la négociatrice FO interviewée :

«Pourquoi est-ce qu'on a été attaqué, nous organisations syndicales, à la sortie de cette convention collective, c'est que dans le cadre de cette réforme y a eu une diminution assez substantielle du montant des frais remboursés, kilométriques, etc. Et en revanche nous ce que nous voulions c'était une vraie professionnalisation du métier, avec du salaire qui donne des droits en matière sociale, de protection sociale et autre. Et donc y a eu un transfert. Mais le transfert n'a pas été à due concurrence.» (16)

Mais la critique du dispositif ne résulte pas seulement de la réaction de «vieux» distributeurs, porteurs d'une mémoire des conditions de travail antérieures à l'institution de la branche. S'il ne s'était agi que de ça, le turn-over et la répression des contestataires auraient pu, à terme, épuiser les sources de critique. Or, la conflictualité sociale est récurrente dans le secteur. Paradoxalement, c'est aussi le statut de distributeur lui-même qui, tout en confortant l'organisation flexible de l'emploi, a permis d'en diffuser la contestation. En objectivant la condition de salarié atypique, il permet aux distributeurs de se penser comme salariés tout en les maintenant dans la pratique aux marges du salariat. Toute une critique profane s'est ainsi développée parmi les distributeurs, qui a souvent croisé une critique experte, menée au nom du droit, notamment par des inspecteurs du travail mécontents de cette entorse au Code du travail.

Dans le monde des distributeurs, certains n'hésitent pas à interpréter le compromis constitutif de la branche comme un échange cynique entre un patronat privilégiant ses intérêts économiques et des représentants syndicaux préoccupés par leurs seuls intérêts d'appareil. Dans cette optique, les distributeurs auraient été une masse de manœuvre mobilisée par les permanents syndicaux pour peser sur la négociation, avant d'être abandonnés à leur sort une fois l'accord conclu. Est également avancé l'argument selon lequel les syndicats auraient été «achetés» avec le mandat de conseiller technique, par lequel le SDD finance un permanent de branche pour chaque syndicat signataire de la convention. Cette lecture trouve d'autant plus de crédit que tous les syndicats reconnus représentatifs dans la branche ont soutenu le compromis. Le divorce entre distributeurs et syndicats est explicitement revendiqué sur le «forum de l'Union des salariés de la distribution directe et des activités postales (Usddap)». Ce réseau internet, créé en 2005, illustre bien l'émergence de formes d'action alternatives aux syndicats. Sur ce site, où l'on dénonce «la complicité active des fédérations syndicales de branches dans l'aide qu'elles apportent et ont apportée aux employeurs,

(16) Entretien, 24 janvier 2012. 
dans l'exploitation des distributeurs et la pratique massive et illégale de travail dissimulé», des distributeurs centralisent des informations juridiques et échangent des conseils pour négocier son contrat de travail ou défendre ses droits face à l'employeur. Une rubrique du forum annonçait même en 2007 l'établissement d'un guide de l'Usddap...

«qui comportera d'une part une évaluation des dépôts, d'autre part une évaluation des délégués du personnel et des délégués syndicaux. Ces informations permettront aux postulants à l'emploi de distributeur ou de messager postal de savoir dans quel dépôt de sa région il est préférable de déposer sa candidature, il permettra aussi aux salariés en fonction de savoir pour qui voter aux diverses élections, du personnel notamment, et qui est à éviter.» (17)

C'est parfois tout simplement une totale extériorité vis-à-vis des syndicats qui s'exprime, comme dans ce courrier d'un distributeur, adressé au Directeur général du travail le 25 janvier 2011, qui demande «que les droits légaux du Code du Travail ne soient plus détournés par des accords entre partenaires». Il est vrai que la fonction de conseiller technique donne quelque réalité à cette représentation d'un monde des "partenaires sociaux» en surplomb, éloigné des distributeurs. Le mandat crée une position d'expert de l'activité de distribution, socialement distincte de l'activité elle-même, qui n'est pas compensée par le profil des syndicalistes quil'investissent : parmi les conseillers actuellement en poste, un seul d'entre eux est ancien distributeur, mais il ne l'était plus depuis longtemps quand il a accédé au mandat (il avait été promu magasinier puis était devenu permanent syndical); un autre est même totalement étranger au secteur puisqu'il est retraité de la Poste.

Relier ces divers phénomènes au rejet des syndicats par les distributeurs s'avère cependant trop schématique. En premier lieu, parce que l'implantation d'un syndicat outsider dans le secteur, Sud-PTT, s'est également nourrie de la critique des organisations syndicales signataires de la CCN. Beaucoup de délégués de cette organisation sont d'ailleurs issus de syndicats concurrents (Unsa, CGT, CFDT, etc.) accusés d'avoir été trop conciliants vis-à-vis des employeurs. Mais les syndicats signataires de la $\mathrm{CCN}$ contribuent également à mettre en forme la critique des salariés mécontents de leur condition. Les syndicats, quelle que soit l'étiquette confédérale, sont en effet les véhicules les plus fréquents des nombreuses contestations prud'homales portant sur la reconnaissance du travail des distributeurs. Ils sont présents dans les grèves qui secouent régulièrement les dépôts sur les problèmes de rémunération ou de conditions de travail. Ainsi, on ne saurait oublier que la formalisation des relations de travail

(17) Source : rubrique du forum de l'Usddap [http:// convdistribution.bbfr.net/forum] consultée le 4 avril 2012, accès réservé aux seuls usagers du forum identifiés. a également conduit à diffuser et légitimer la forme syndicale, de manière à la fois directe et indirecte. À travers la signature de la $\mathrm{CCN}$, le patronat du secteur a reconnu la légitimité d'une représentation syndicale qui, loin de se limiter au niveau de la branche, est censée s'articuler étroitement à une présence syndicale renforcée dans les entreprises. Ainsi, dans la foulée de la CCN sont négociés en 2005, au sein des entreprises, des accords ou avenants qui actent des nouvelles règles en matière de droit syndical. Il s'agit en particulier d'appliquer le coefficient multiplicateur qui permet d'établir le nombre de mandats représentatifs. À Distrib, l'entreprise est conduite à redéfinir son rapport aux syndicats, comme en témoigne un responsable de l'encadrement :

«Y avait quand même une image négative du syndicat. Même moi j'avais aussi cette image... [...] La convention a amené beaucoup. Je sais pas si c'est le fait d'écrire, des droits et des obligations des employés... c'est monté en puissance sur le social. [...] Il a fallu former le réseau aux relations sociales. »(18)

La CCN a par ailleurs accompagné - et sans doute accéléré - un mouvement de réorganisation économique du secteur qui a également contribué à la diffusion de la présence syndicale. Parallèlement à l'entrée sur le marché du nouvel opérateur Gratuit, son principal concurrent Distrib avait engagé une stratégie d'acquisitions de sociétés dont certaines disposaient déjà d'une représentation syndicale, notamment dans le sud-ouest de la France. Le marché de la distribution directe était en fait un front avancé dans la guerre que préparaient les deux principaux opérateurs sur fond de libéralisation du marché postal ${ }^{(19)}$. L'exacerbation de la concurrence a conduit à la constitution d'un quasi-duopole, par rachats successifs et liquidation des entreprises les moins compétitives, tandis qu'au sein de chaque entreprise les injonctions à la rentabilité économique se faisaient plus fortes. C'est autant l'institution de la $\mathrm{CCN}$ que l'intégration de structures de dimension locale à des entités nationales qui ont progressivement fait passer les relations de travail du mode domestique au mode bureaucratique, disqualifiant - et rendant parfois techniquement impossibles (20) - les arrangements informels localisés. Parmi les militants rencontrés, plusieurs ont justifié leur engagement syndical, au cours des années 2000, par une détérioration du climat sur leur lieu de travail imputable à ces transformations.

(18) Entretien, 7 décembre 2010.

(19) Dans le contexte d'ouverture du marché postal, Distrib décide à l'époque d'investir le secteur du courrier adressé. En 2006, l'entreprise devient le premier opérateur privé à acquérir la «licence postale». Bien que Distrib ait finalement renoncé à concurrencer la Poste sur un marché totalement libéralisé

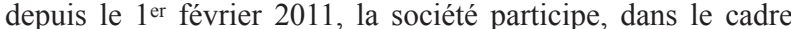
du Syndicat des opérateurs postaux, aux négociations devant déboucher sur une convention collective des activités postales. (20) Le calcul des rémunérations est par exemple davantage cadré par l'introduction de logiciels informatiques. 
En outre, en même temps qu'elle créait les conditions pour une plus grande implication syndicale des salariés, la CCN le faisait sur la base d'un rapport utilitariste aux syndicats, en confortant un intérêt à la syndicalisation relevant moins de la défense des «acquis» de la convention que du souci d'obtenir des avantages pour soi. Les témoignages abondent, de la part de militants syndicaux variés, au sujet de représentants syndicaux ou d'élus du personnel sans aucune «conscience syndicale». Dans une logique analogue à celle qui existe dans l'intérim, la décharge syndicale vient en effet compléter la rémunération initiale (Grollier, 2010). Les mandats peuvent ainsi être utilisés par les distributeurs comme une «prime», voire comme un coût infligé à l'entreprise, afin de faire monter les enchères au moment de négocier un licenciement (et, après 2008, une rupture conventionnelle) ou d'inciter l'employeur à proposer un avancement. Ils peuvent également être proposés comme récompense par l'employeur lui-même. Ces usages, qui sont déviants au regard de la norme militante légitime du désintéressement, se comprennent dans la configuration spécifique, à la fois sociale et juridique, qui les informe : alors même que le temps partiel et, avec lui, précarité et bas salaires continuent de dominer le secteur, la CCN a institué un relatif découplage entre l'ouverture des droits salariaux et celle des droits syndicaux, tandis que l'absence de collectifs de travail et la personnalisation des rapports avec l'encadrement de proximité facilitent les stratégies individuelles. En outre, les entretiens menés avec des distributeurs syndiqués montrent à quel point les investissements intéressés et «l'intérêt au désintéressement» (BouRdieu, 1984) s'entremêlent et se redéfinissent continûment dans le cours de la carrière syndicale.

L'institutionnalisation du secteur par la convention de branche n'a donc pas eu pour conséquence de couper les syndicats des distributeurs. Elle a plutôt eu deux effets en partie contradictoires : d'une part, favoriser la diffusion de la forme syndicale en tant que ressource juridique et son appropriation, selon des logiques diverses, au sein des entreprises; d'autre part, stabiliser une arène de représentation supplémentaire ayant sa logique propre, celle de la branche. Cette reconfiguration a créé une tension structurelle, tension qui n'oppose pas seulement les distributeurs aux représentants syndicaux, mais qui traverse également tous les syndicats présents sur les deux scènes. Le paysage de la distribution directe, tel que l'a mis en forme la CCN, donne ainsi l'image d'un arrangement instable, que la réforme de 2008 pourrait avoir définitivement ébranlé.

\section{Les dynamiques contradictoires de la "démocratie sociale" : ce que la réforme de 2008 a changé}

La réforme de 2008 accorde aux élections professionnelles une importance qu'elles n'avaient pas dans la configuration antérieure. En subordonnant la représentativité légale des syndicats à l'expression élective des salariés, elle vise explicitement, au nom de la «démocratie sociale», à rapprocher les deux dimensions de la représentativité (légale et sociale) évoquées plus haut. On a vu précédemment que les règles de droit se prêtent à des usages pluriels; elles ne sauraient donc avoir des effets mécaniques. La façon dont sont mobilisées les nouvelles règles de représentativité dans les deux principales entreprises de la branche en témoigne. En deçà des usages, la réforme de 2008 implique cependant une reconfiguration de l'ordre juridique qui met un terme à la déconnexion structurelle entre représentation d'entreprise et représentation de branche.

\section{Une syndicalisation ambivalente dans les entreprises}

La réforme de la représentativité syndicale a conforté la tendance à la syndicalisation des relations de travail qui avait été impulsée par l'institutionnalisation de la branche. C'est particulièrement visible dans le cas de Distrib. Tout en concédant des moyens nouveaux aux organisations syndicales, l'entreprise maintenait, aux dires des syndicalistes interviewés, une politique d'«endiguement» du fait syndical. Celle-ci se traduisait notamment par la promotion de candidats non syndiqués aux élections de délégués du personnel (DP) et aux élections du comité d'entreprise (CE), qui débouchait sur la «neutralisation» des instances représentatives du personnel (IRP). La proximité supposée entre la majorité du $\mathrm{CE}$ et la direction permettait à celle-ci de ne pas rencontrer d'obstacle dans les démarches nécessitant l'avis du comité, qu'il s'agisse des licenciements de salariés protégés, du suivi des typologies de préquantification ou des orientations économiques et sociales de l'entreprise. À l'occasion du renouvellement des IRP, en 2010, l'entrée en vigueur de la loi de 2008 change la donne. On assiste au basculement de candidats auparavant non syndiqués sur les listes d'un syndicat autonome, la Confédération autonome du travail $(\mathrm{CAT})^{(21)}$. Son fondateur dans l'entreprise reconnaît en avoir eu l'idée après une réunion de formation organisée par la direction et animée par des consultants extérieurs à l'entreprise (22). La suppression de la présomption irréfragable de représentativité a en outre permis à

(21) Cette organisation peu connue revendique une existence depuis 1953. Elle dispose d'un site internet : http://www.c-a-t.

(22) Entretien, 7 décembre 2010. 
Sud-PTT de participer à la négociation du protocole d'accord préélectoral et de s'implanter(23). Quelques représentants de section syndicale (RSS) furent désignés avant les élections, mais uniquement dans les dépôts où existaient des chances de les faire élire ${ }^{(24)}$. Toutes les organisations syndicales progressent en nombre de voix et étendent leur implantation, ce qui témoigne des efforts fournis face à l'enjeu nouveau de représentativité. L'augmentation de la participa-

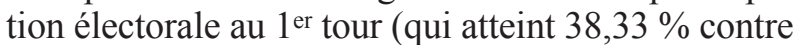
$17,05 \%$ au précédent scrutin de 2007) et la baisse du nombre de bulletins blancs et nuls (de 18,38\% à $10,31 \%$ ) peuvent ainsi s'expliquer doublement : ce sont non seulement les syndicats, mais aussi la direction d'entreprise qui furent intéressés à l'issue du scrutin. Dans un contexte où les injonctions plus ou moins contraignantes à la négociation d'entreprise se multiplient, le fait que les nouvelles règles subordonnent le pouvoir de signature des syndicats à leur représentativité électorale aurait incité, d'après certains interviewés, l'employeur à miser sur un syndicat autonome au 1er tour plutôt que sur un $2^{e}$ tour ouvert aux non-syndiqués. Avec 13,4 \% des suffrages exprimés à l'issue du 1er tour, la CAT gagne la représentativité. Sud n'atteint pas le seuil des $10 \%$, mais en s'approchant des $8 \%$, ce syndicat obtient un stock de voix qui lui permet de prétendre à la représentativité au niveau de la branche. Pour la première fois dans l'histoire de l'entreprise, une majorité syndicale conquiert le comité d'entreprise qui, dans la foulée, active un certain nombre de prérogatives juridiques restées en sommeil jusqu'alors (notamment en matière d'expertise économique).

La situation est plus complexe à Gratuit, où la présence syndicale était devenue un fait établi, via la présence de nombreuses organisations : CGT, CFDT, FO, CFTC, CFE-CGC (Confédération française de l'encadrement - Confédération générale des cadres), Unsa, Sud-PTT. L'opérateur public a importé une rhétorique du «dialogue social» et un savoir-faire en matière de gestion des relations collectives de travail tranchant avec la politique antisyndicale qui prévalait jusqu'alors dans le secteur : «Voter est un acte de "citoyen d'entreprise" qu'il faut encourager afin que s'exprime pleinement la démocratie de l'entreprise», pouvait-on déjà lire dans le protocole d'accord préélectoral de 2007. L'effort fourni en matière d'organisation électorale avait à l'époque permis d'accroître considérablement le taux de participation, passé de moins de 30 à

(23) C'est à l'occasion de l'entrée de Gratuit, propriété du groupe La Poste, sur le marché de la distribution que Sud-PTT, qui développe une stratégie de groupe, a pris pied dans la distribution directe. Le syndicat avait réussi à imposer sa représentativité au sein de Gratuit malgré l'opposition de l'entreprise et pu présenter des listes au $1^{\text {er }}$ tour des élections professionnelles dès 2007 .

(24) En effet si, à l'issue des élections, le syndicat que représente un RSS n'a pas atteint le seuil de représentativité, un autre salarié doit être désigné RSS et le statut de salarié protégé disparaît au bout de six mois.
$49,9 \%$ entre les élections de 2005 et 2007 . Il atteint $62,2 \%$ au scrutin suivant de 2011. La direction a l'habitude de négocier - «en 5 ans j'ai dû signer 36 accords», me dit l'actuel directeur des ressources humaines ${ }^{(25)}$ - et dispose de partenaires privilégiés, variables selon les enjeux, mais où se retrouvent souvent deux «petits » syndicats l'Unsa et la CFTC. Dans une entreprise où le dialogue social est installé et célébré, l'hostilité vis-à-vis de l'employeur ne peut servir de liant et c'est moins la coopération que la concurrence entre syndicats qui semble prévaloir. Pendant plusieurs années, un syndicat CGT d'entreprise a existé concurremment à une représentation cégétiste fédérale, traduction organisationnelle de la tension structurelle entre les niveaux de la branche et de l'entreprise. Lors des élections professionnelles d'octobre 2011, des listes Sud-Gratuit sont venues concurrencer les listes Sud-PTT. Cette dissidence a été facilitée par la structure des IRP qui, à la différence de Distrib, est décentralisée. Tous les syndicats ne se sont pas présentés dans les six CE et tous n'ont pas atteint le seuil de représentativité. Les élections de 2011 ont ainsi débouché sur une représentativité syndicale à géométrie variable. $\mathrm{La}$ CFDT et le CGT sont les deux seules organisations représentatives dans tous les CE. Viennent ensuite FO, la CFTC et Sud-PTT qui sont respectivement représentatives dans cinq, quatre et trois CE sur six. L'Unsa est la grande perdante, qui n'est reconnue représentative que dans un seul établissement et perd sa représentativité au niveau de l'entreprise nationale, ce qui prive la direction d'un interlocuteur privilégié dans la négociation d'entreprise. Sud-Gratuit, qui avait présenté des listes dans deux régions, n'obtient la représentativité à aucun niveau.

Les nouvelles règles de représentativité ont autorisé divers «coups» juridiques, de la part des organisations syndicales ou des employeurs. Dans le protocole d'accord concernant les élections DP, la direction de Distrib a par exemple introduit une clause excluant les chefs de dépôt du corps électoral. En privant ces derniers de l'onction électorale, elle coupait l'herbe sous le pied des syndicats qui, telle la CFTC, avaient entrepris de les syndiquer en désignant certains d'entre eux délégués syndicaux. De même, Sud-PTT a réussi à porter un coup fatal à la CGC en contestant jusque devant la Cour de cassation l'identité catégorielle de cette organisation. Au $1^{\text {er }}$ tour du 8 octobre 2010, le syndicat CGC n'avait présenté de candidats que dans les deuxième et troisième collèges et fait reconnaître sa représentativité sur cette base catégorielle. Le quorum n'ayant pas été atteint, un deuxième tour était organisé le 19 novembre 2010 lors duquel la CGC présenta cette fois des listes dans les trois collèges. Croisant ce fait avec les statuts du syndicat où il est inscrit qu'il représente «sous certaines conditions,

(25) Entretien, 18 janvier 2012. 
les employés», la Cour en a déduit, dans une décision du 31 janvier 2012, que la représentativité de la CGC devait être mesurée tous collèges confondus, ce qui eut pour conséquence de faire perdre au syndicat sa représentativité et d'annuler la désignation de son délégué syndical central. Sur le site de la section FO-Distrib, il est écrit à ce sujet : «Alors que la direction attaque systématiquement quand elle le peut les désignations des syndicats employés, pour le syndicat des cadres il aura fallu que ce soit le syndicat Sud qui attaque pour que justice soit faite.»

À Gratuit, la subdivision de la représentation professionnelle en $\mathrm{CE}$ de région datait du scrutin de 2007. La Direction du travail avait répondu à une demande d'arbitrage de la CGT en imposant cette décentralisation. Un mois plus tard, la direction de Gratuit dénonçait le chapitre de l'accord d'entreprise de 2005 consacré au droit syndical : elle assortissait ses propositions de régionalisation du droit syndical d'une réduction de certains moyens, ce qui devait aboutir à la signature d'un «Accord sur le dialogue social au sein de Gratuit», en janvier 2009, par trois syndicats seulement (CFTC, FO et Unsa). Une clause de l'accord, d'apparence anodine à l'époque, devait avoir d'importantes conséquences :

«Les dispositions sur les DS s'appliqueront jusqu'aux prochaines élections dans tous les CE. Elles cesseront de s'appliquer de plein droit après la proclamation des résultats de ces élections afin d'être en conformité avec la loi du 20 août 2008 pour la partie portant sur la rénovation de la démocratie sociale. Une nouvelle négociation s'engagera dans les six mois précédant la date du 1 er tour. »»

La négociation fut cependant engagée après les élections. D'après un délégué syndical central interviewé, «le fait de négocier avant les élections aurait permis aux organisations syndicales de prévoir, si elles n'étaient pas représentatives, ce qui se passerait pour elles. Ce que la direction ne voulait pas, parce que ça engendrerait des dépenses»(26). Ce report de la négociation eut d'autres conséquences suite à la jurisprudence du 10 novembre 2010. Dans cette décision, la Cour de cassation fait du CE et non des DP le périmètre par défaut pour la désignation des DS (27). Concrètement, cela impliquait à Gratuit la disparition de DS de plateforme, le niveau de représentation CE étant la région ${ }^{(28)}$. Suite aux élections, la direction obtint ainsi l'annulation de la plupart

(26) Entretien, 5 octobre 2012.

(27) Dans plusieurs arrêts du 10 novembre 2010, confirmés par un arrêt du 18 mai 2011, la chambre sociale de la Cour de cassation a opéré un revirement de jurisprudence : elle a aligné sa définition de la notion d'établissement distinct, cadre de désignation du délégué syndical, sur celle, formulée par le Conseil d'État, de l'établissement distinct au sens de l'élection du comité d'établissement.

(28) Si le CE est national à Distrib, l'accord d'entreprise qui organise la désignation des délégués syndicaux par établissement d'au moins 50 salariés en équivalents temps plein est toujours en vigueur. des désignations de DS d'établissement devant les tribunaux d'instance (29), tout en proposant à la négociation un projet d'accord qui reconnaissait comme périmètre de désignation «l'établissement distinct entendu au sens du comité d'établissement.» Dans un communiqué commun, en novembre 2011, les syndicats accusaient la direction de vouloir «supprimer les délégués syndicaux sur toutes les plateformes pour isoler encore plus les salariés et éloigner les organisations syndicales des lieux de travail»(30). Malgré l'opposition d'une majorité d'organisations, un accord a été conclu le 7 juin 2012 avec $\mathrm{FO}$, la CGC et la CFTC, qui représentent à peine plus de la majorité relative de $30 \%$ requise $(32,8)$. Paradoxalement, l'entrée en vigueur de la réforme à Gratuit a donc conduit à une restriction de la représentation syndicale. En 2010, d'après le bilan social de l'entreprise, 393 délégués syndicaux avaient été effectivement désignés selon les règles en vigueur à l'époque. Selon le texte signé en 2012, en fonction de la configuration d'organisations représentatives, le maximum de délégués désignables, toutes organisations confondues, est ramené à $179^{(31)}$.

La comparaison des deux entreprises permet ainsi de nuancer l'hypothèse selon laquelle la réforme serait un point d'appui pour le développement syndical. Sur ce point, la question des mandats est cruciale : le premier pas vers l'engagement est généralement l'obtention d'un mandat électif ou désignatif, parce qu'il offre une protection légale. C'est pourquoi les collectifs syndicaux actifs se résument le plus souvent aux salariés mandatés. Dans les secteurs précaires, la crainte des représailles de l'employeur est d'autant plus forte qu'elles peuvent être à la fois plus dramatiques et moins visibles. Or, l'onction électorale requise pour les DS restreint le vivier des désignations. Elle empêche un usage du mandat DS comme mandat d'implantation, que la création du mandat de représentant de section syndicale (RSS) ne compense que partiellement : outre le fait que celui-ci dispose de moins de moyens, il permet de s'implanter uniquement dans les périmètres où une organisation n'a pas fait la preuve de sa représentativité. Concrètement, il est impossible pour la CGT, FO, la CFDT, la CFTC et la CAT, tous représentatifs à Distrib, de désigner des délégués syndicaux dans les établissements où ils n'auraient pas préalablement présenté de candidats aux élections. Paradoxalement, seuls Sud-PTT, la CFE-CGC ou de nouveaux outsiders pourraient s'implanter en désignant des représentants de section syndicale. À Gratuit, la contradiction a été résolue dans un sens défavorable à la représentation

(29) Seuls quatre jugements ont été favorables aux syndicats et doivent passer en cassation.

(30) «Préalable à toute négociation», déclaration de l'intersyndicale Gratuit CFDT-CFTC-CGT-FO-Sud, novembre 2011.

(31) Ces données doivent encore évoluer en 2013 suite à un projet de réorganisation des périmètres de gestion de l'entreprise. 
syndicale, l'employeur s'appuyant sur la jurisprudence qui ramène le périmètre de désignation des DS à celui des CE. La centralité donnée au critère de l'audience électorale dans la détermination de la représentativité a d'abord pour conséquence de légitimer une définition de l'activité syndicale centrée sur les arènes de dialogue social plutôt que sur les lieux de travail. Dans le secteur de la distribution directe, ce changement a pour effet d'éloigner un peu plus la réalité du secteur du schéma idéal qui avait présidé au compromis de branche : rappelons que l'acceptation du statut dérogatoire pour les distributeurs était conditionnée à la mise en place d'instances de suivi au niveau de la branche (conseillers techniques) et des établissements (IRP), la connexion entre ces deux niveaux devant être assurée par le canal syndical. En outre, le nouveau cadre juridique conduit à indexer davantage les conditions de la représentation syndicale aux accords collectifs (protocoles préélectoraux et accords de droit syndical) qui organisent, dans chaque entreprise, les périmètres de représentation du personnel. Il en résulte que les «effets» de la loi sont d'autant moins saisissables hors des configurations propres à chaque entreprise, comme l'illustre le paradoxe d'une restriction de la présence syndicale dans l'entreprise supposée la plus ouverte au dialogue social, tandis que la syndicalisation de l'entreprise perçue comme la plus antisyndicale se trouve encouragée.

\section{Vers une déstabilisation finale du compromis de branche?}

À défaut de renforcer la présence syndicale, les nouvelles règles engendrent-elles un resserrement du lien représentatif? Que la démocratie sociale intervienne en substitut à une démocratie syndicale défaillante était l'effet le plus direct et attendu de la réforme, comme moyen d'accroître le contrôle des salariés sur leurs représentants. En réalité, le contrôle est moins direct qu'indirect, via le pouvoir de sanction que donne aux électeurs le droit de ne pas reconduire les sortants. Il est donc impossible à mesurer dans le cadre d'un seul cycle électoral. Mais l'ampleur du turn-over parmi les salariés de la distribution directe, à l'opposé de la tendance à l'accroissement de la durée des mandats (elle est maintenant de quatre ans, sans compter les prorogations consécutives aux désaccords lors des négociations des protocoles préélectoraux), rend peu probable l'instauration d'une telle contrainte sur l'action des représentants. Par ailleurs, l'exercice de ce contrôle supposerait qu'existe une "sphère de publicité» (HABERMAS, 1993) permettant aux salariés de prendre connaissance des dossiers abordés dans les arènes de dialogue social et de les soumettre à la critique argumentée. L'importance nouvelle accordée aux élections a-t-elle par exemple donné une meilleure visibilité à la question controversée du statut de distributeur? A-t-elle permis aux distributeurs de départager en connaissance de cause les syndicats en fonction de leurs positions sur le sujet? Les chances que les électeurs se saisissent de tels enjeux revendicatifs dépendent de la façon dont les acteurs syndicaux eux-mêmes construisent leur campagne. À consulter les syndicalistes et leur matériel électoral, le statut des distributeurs est au mieux une question parmi d'autres, et sans doute la moins concrète. Il apparaît comme le fond sur lequel se jouent des enjeux plus immédiats : les transformations du métier liés à la mécanisation, les conditions de travail ou les faibles rémunérations. En outre, l'impact direct des élections au comité d'entreprise concerne d'abord, pour les salariés, les actions menées dans le domaine des activités sociales et culturelles, et une partie de la campagne se fait sur ce point. Ces limites sont inscrites dans le décalage entre les procédures qui organisent le rapport social de représentation et les enjeux auxquels il est censé répondre : décalage entre, d'une part, la fonction tangible du CE, le cadre d'organisation des élections (l'entreprise, voire les établissements) et, d'autre part, les enjeux du dialogue social, dont les plus décisifs se jouent dans la branche. Dans la mesure où les élections, qui déterminent désormais le paysage syndical pertinent, se déroulent dans les entreprises autour d'enjeux multiples, l'arène paritaire continue d'œuvrer au «confinement des espaces de circulation des controverses et des débats » et à différencier les acteurs «responsables», intégrés à ce forum, des «irresponsables» extérieurs à lui (HENRY, 2005).

Ce sont ainsi moins les campagnes électorales que les contentieux juridiques qui contribuent à publiciser l'enjeu du statut des distributeurs, en particulier la dénonciation de décrets du ministère du Travail qui, par deux fois, ont cherché à sécuriser juridiquement le système de préquantification ${ }^{(32)}$. Mais ces actions sur le terrain du droit, si elles ont permis de mettre en visibilité la condition précaire des distributeurs, n'ont pas pour autant conduit à une clarification des positionnements syndicaux. En même temps qu'ils accompagnent la critique du statut des distributeurs au sein des entreprises, les syndicats représentatifs au niveau de la branche ne s'en désolidarisent jamais totalement sur la scène paritaire. Ainsi, la seule organisation syndicale signataire de la $\mathrm{CCN}$ qui a dénoncé le décret de 2010 (FO) ne l'avait pas fait au nom de l'illégalité du dispositif (contrairement à Sud) mais au nom de la «mauvaise application» de «bonnes règles» ${ }^{(33)}$. Quant à la CGT, si elle se prononce désormais dans ses tracts pour un retour au contrôle effectif du temps de travail, «à partir d'auto-déclaratifs ou de systèmes de contrôle de travail activés

(32) Un premier texte, paru en 2007, a été dénoncé par Sud-PTT et censuré par le Conseil d'État dans un arrêt du 11 mars 2009; un second texte, adopté en 2010, a été dénoncé par Sud et FO et de nouveau censuré dans une décision du Conseil d'État du 28 mars 2012

(33) Le Conseil d'État a donné raison à Sud en rappelant que seule une loi pourrait autoriser un système de rémunération du travail à se passer de contrôle du temps de travail effectif. 
Tableau : Résultats électoraux des organisations syndicales présentes dans la branche

\begin{tabular}{|c|c|c|c|}
\hline & $\begin{array}{c}\text { Distrib } \\
\text { (scrutin du } 8 \text { octobre 2010) }\end{array}$ & $\begin{array}{c}\text { Gratuit } \\
\text { (scrutins du } 10 \text { au } 14 \text { octobre } \\
2011 \text { et du } 4 \text { mai 2012) }\end{array}$ & Résultats agrégés \\
\hline Effectif & 20511 & 13215 & 33726 \\
\hline Votants & $7851(38,28 \%)$ & 1 & 1 \\
\hline Suffrages exprimés & $6934(33,81 \%)$ & $8158(61,73 \%)$ & $15392(45,64 \%)$ \\
\hline CGT & $1672(\mathbf{2 4 , 1 1} \%)$ & $1838(22,53 \%)$ & $3510(22,80 \%)$ \\
\hline CFDT & $1034(\mathbf{1 4 , 9 1 \% )}$ & $2015(\mathbf{2 4 , 7 0} \%)$ & $3049(19,81 \%)$ \\
\hline CGT-FO & $1659(23,93 \%)$ & $1334,13(\mathbf{1 6 , 3 5} \%)$ & $2993,13(19,45 \%)$ \\
\hline CFTC & $793(\mathbf{1 1}, \mathbf{4 4} \%)$ & $1091(\mathbf{1 3 , 3 7} \%)$ & $1884(12,24 \%)$ \\
\hline Unsa & $242(3,49 \%)$ & $524,44(6,43 \%)$ & $766,44(4,98 \%)$ \\
\hline Sud-PTT & $540(7,79 \%)$ & $945,43(\mathbf{1 1 , 5 9} \%)$ & $1485,43(9,65 \%)$ \\
\hline CFE-CGC & $\begin{array}{r}65(0,94 \%) \\
\left(\mathrm{AM}^{*} / \text { cadres : } 33,51 \%\right)\end{array}$ & $\begin{array}{r}285(3,49 \%) \\
(\mathrm{AM} / \text { cadres : } \mathbf{3 5 , 3 2} \%)\end{array}$ & $\begin{array}{r}350(2,27 \%) \\
(\mathrm{AM} / \text { cadres : } 34,96 \%)\end{array}$ \\
\hline CAT & $929(\mathbf{1 3 , 4 0} \%)$ & 1 & $929(6,04 \%)$ \\
\hline Sud Gratuit & 1 & $125(1,53 \%)$ & $125(0,81 \%)$ \\
\hline
\end{tabular}

* AM : agents de maîtrise.

Lecture : En gras les résultats pertinents pour la représentativité légale.

La représentativité catégorielle de la CFE-CGC à Distrib a été annulée par la Cour de cassation. Les résultats à Gratuit ont été recalculés après annulation des élections dans le collège Employés de l'établissement Bourgogne-Rhône-Alpes (BRA). De nouvelles élections ont été organisées dans ce seul collège en mai 2012. La présence de décimales au nombre de suffrages décomptés pour FO, Unsa et Sud-PTT à Gratuit s'explique par le fait que ces syndicats ont présenté une liste unitaire dans l'établissement BRA, les suffrages ayant été divisés selon une clé de répartition indiquée par les organisations. Concernant la branche, les résultats sont indicatifs car ils ne couvrent pas la totalité des salariés couverts par la CCN.

Source : données calculées par l'auteur sur la base des résultats arrêtés dans les entreprises.

par [les salariés] en début et en fin de tournée»(34), elle n'a jusqu'alors pas tenté de dénoncer la CCN ni attaqué le décret devant le Conseil d'État. Si la décision de la haute juridiction a conforté symboliquement la position de Sud et de la CGT, conduisant FO à avancer l'idée d'un contrôle effectif du temps de travail par géolocalisation, elle n'a pas pour autant mis un terme aux négociations de révision de la CCN dans le même cadre dérogatoire. Le SDD va jusqu'à évoquer la nécessité de faire changer la loi, éventualité que certains syndicats n'écartent pas. La décision du Conseil d'État est cependant encore trop récente et des réorientations syndicales pourraient encore en découler. Le changement de majorité parlementaire et, avec lui, une inflexion des politiques du travail, pourraient également modifier les rapports de force à court terme.

Au-delà de ces incertitudes conjoncturelles, la réforme de la représentativité syndicale pourrait cependant avoir comme effet de dénouer la tension structurelle en déstabilisant définitivement le compromis de branche. Au niveau des branches professionnelles, l'ancien régime de représentativité était typique de la logique du «partenariat social» : il combinait la présélection par l'État (c'était la présomption irréfragable des cinq confédérations «historiques») et la reconnaissance mutuelle (c'est la signature d'une $\mathrm{CCN}$ qui constitue une branche, et c'est pour signer une $\mathrm{CCN}$ que se constituent des acteurs, notamment patronaux). Or, en faisant

(34) «Temps de travail : le gouvernement passe en force!», tract FILPAC-CGT, juillet 2010. reposer la représentativité syndicale de branche sur le vote des salariés plutôt que sur la reconnaissance de l'interlocuteur patronal, le nouveau dispositif déconnecte en partie la représentativité de la logique du partenariat social, tout en connectant l'arène paritaire aux entreprises. La probabilité d'une évolution du paysage syndical représentatif à ce niveau pourrait remettre en cause le compromis constitutif de la branche, inscrit dans la CCN. L'acceptation du statut dérogatoire des distributeurs avait en effet pour contrepartie, on l'a vu, une forte reconnaissance institutionnelle des syndicats. Or, les nouvelles règles de représentativité tendent à dissocier les termes de cet échange : sur la base d'une représentativité conquise par les urnes, des acteurs syndicaux qui rejettent le statut dérogatoire des distributeurs, tel Sud-PTT, seront conduits à exiger le bénéfice des droits syndicaux inscrits dans la $\mathrm{CCN}$; en parallèle, les acteurs syndicaux menacés dans leur représentativité pourraient être incités à reconsidérer leur attitude face au système. Les partenaires sociaux ont jusqu'à maintenant repoussé ces épreuves fatidiques. Au lendemain des élections professionnelles à Distrib (voir tableau), Sud-PTT avait interpellé les partenaires sociaux pour être invité aux réunions paritaires et, parallèlement, demandé à la DGT d'engager une enquête de représentativité. Sans réponse, le syndicat avait réitéré sa demande à l'automne 2011, suite au renouvellement des IRP à Gratuit. S'appuyant sur le texte de 2008 qui prévoit que la liste des organisations représentatives soit établie à l'issue d'un cycle électoral, le SDD et les syndicats représentatifs selon l'ancien mode ont reporté leur décision à 
2013. Parallèlement, la perte de représentativité de la CGC à Distrib pourrait provoquer une réaction en chaîne lui faisant perdre le bénéfice de la représentativité catégorielle au niveau de la branche entière.

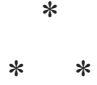

Au-delà de sa question de départ - la loi de 2008 contribue-t-elle à resserrer le lien entre les salariés et leurs syndicats ? - ce texte a cherché à mettre en doute un motif central dans les discours de justification de la réforme, mais aussi dans la plupart des analyses du syndicalisme : le problème des syndicats est-il réellement celui de leur excessive institutionnalisation? À l'encontre des lectures courantes qui associent «institutionnalisation» et «désyndicalisation», un retour sur la genèse de la branche de la distribution directe permet de voir que l'institutionnalisation produit aussi de la syndicalisation. L'entrée dans un processus de négociation institutionnalisée au niveau de la branche professionnelle, «par en haut», loin de couper les syndicalistes de leur base, a au contraire eu pour conséquence de précipiter l'implantation syndicale "sur le terrain", dans les entreprises et les établissements. Elle a contraint les syndicats à mobiliser des salariés du secteur et pesé, en retour, sur les négociations de branche et d'entreprise. Mais pour que les syndicats puissent ainsi être constitués en représentants des distributeurs, il fallait que les formes du travail de distributeur soient elles-mêmes modifiées. L'institution de la $\mathrm{CCN}$ a reconfiguré le rapport des distributeurs à leur propre travail autant que leur rapport au syndicalisme. Observer la condition syndicale par la focale élargie de la formalisation des relations de travail permet ainsi de considérer l'institutionnalisation du syndicalisme dans sa dynamique contradictoire. Que les relations de travail soient «saisies par le droit» ne signifie pas qu'elles sont pétrifiées par lui. Le droit ne «cause» pas les pratiques, il les oriente comme «un des référents sur la base desquels se construisent les interactions» (Lascoumes, Serverin, 1995, p. 165). La représentativité légale ne peut donc pas être considérée comme une variable extérieure qui permettrait d'intervenir sur la représentativité sociale des syndicats. Elle constitue plutôt une ressource que différents acteurs - les syndicalistes eux-mêmes, mais aussi les employeurs, les agents de l'État, etc. - mobilisent selon des logiques plurielles.

Une telle mise en perspective permet d'étudier l'impact de la réforme de la représentativité syndicale de manière plus nuancée, sans céder à la dénonciation ou aux louanges a priori. La mobilisation des règles de représentativité dans les deux entreprises montre que les effets de la réforme en termes de syndicalisation s'avèrent ambivalents. D'abord parce que le nouveau régime de représentativité doit être internalisé dans les conditions propres à chaque configuration d'entreprise pour révéler ses effets. Il apparaît alors qu'il n'apporte pas nécessairement de nouvelles ressources pour les organisations syndicales. Il peut même impliquer de devoir faire mieux avec autant, sinon moins de moyens. Ensuite, parce que la procédure faisant découler la représentativité syndicale des élections professionnelles, loin d'être neutre, constitue l'acteur syndical à partir des arènes du dialogue social. Dans le cas de la distribution directe, cela engendre des contraintes nouvelles, différentes selon l'entreprise, en termes d'implantation syndicale sur les lieux de travail. La loi agit ainsi comme un révélateur de la fragilité des syndicats plutôt que comme un levier de leur renforcement. À défaut de garantir ce renforcement du syndicalisme, la réforme de la représentativité permet-elle un contrôle plus étroit des salariés sur leurs représentants? On l'a vu, la réforme a déjà commencé d'ébranler le compromis constitutif de la branche. Mais la déstabilisation de l'arrangement de branche ne relève pas d'un arbitrage des salariés en faveur des organisations qui lui sont hostiles. Elle ne relève pas non plus d'un contrôle accru des mandataires par leurs mandants, ni d'une discussion collective plus transparente des enjeux de la branche : les nouvelles règles de représentativité n'impliquent aucun décloisonnement des questions mises en délibération. Elles apportent en revanche un imprévu lié à la nouvelle mécanique juridique, en tant qu'elles connectent des procédures désignatives auparavant disjointes.

Les nouvelles règles de représentativité répondent donc d'autant moins au «problème» de l'institutionnalisation des syndicats que ce diagnostic est mal formulé. Il semble plus juste de considérer la réforme comme un dispositif reconfigurant l' $l^{\prime}$ institutionnalité» syndicale. Cette reformulation permet d'en finir avec la vision strictement négative qui sous-tend la thèse de l'institutionnalisation ${ }^{(35)}$ et qui laisse entendre qu'il pourrait exister hors des institutions, hors du droit, un syndicalisme vivant, libéré de toute forme pétrifiée (36). C'est aussi la limite d'une analyse critique informée par le marxisme d'Althusser, qui mobilise la dichotomie base/sommet (Higelé, 2012). L'enjeu est donc moins de chercher à «contrer» ou «limiter» l'institutionnalisation du syndicalisme que de distinguer les différentes formes d' «institutionnalité» qui le traversent. On pourrait ainsi, avec Michels (1971), se fixer la tâche

(35) Une autre entrée dans la critique de l'institutionnalisation, par la méthode ethnographique, est proposée par Julian Mischi (2011) qui montre qu'à la «base», le rapport entre salariés et représentants syndicaux relève moins de la simple coupure que d'arbitrages permanents entre distance et proximité.

(36) ...ou pas : ainsi Pierre Rosanvallon, (1998, p. 247) concluait-il sa réflexion en pointant cette contradiction : «Le syndicalisme est ballotté entre une légitimation de nature sociologique, désormais insuffisante, et une légitimation de nature politique, impossible». 
de repérer les prises susceptibles «de fortifier et d'exciter chez l'individu l'aptitude intellectuelle à la critique et au contrôle» (p. 301), afin de conforter ce qu'il appelait les «tendances démocratiques» face aux «tendances oligarchiques». Une telle perspective permettrait en outre d'opérer la jonction avec les définitions sociologiquement plus robustes de l'institutionnalisation, qui ne réduisent pas ce concept à sa dimension juridique mais considèrent l'ensemble des normes qui orientent les pratiques sociales.

\section{Bibliographie}

AdAm G. (1983), Le pouvoir syndical, Paris, Dunod.

Andolfatto D., LabBé D. (2006), Histoire des syndicats (1906-2006), Paris, Seuil.

Andolfatto D., LaBBÉ D. (2009), Toujours moins! Déclin du syndicalisme à la française, Paris, Gallimard.

Bergounioux A. (1975), Force Ouvrière, Paris, Seuil.

Béroud S., Bouffartigue P. (dir.) (2009), Quand le travail se précarise, quelles résistances collectives?, Paris, La Dispute.

Bévort A., Jobert A. (2008), Sociologie du travail : les relations professionnelles, Paris, Armand Colin.

Borenfreund G., Souriac M.-A. (dir.) (2008), Syndicats et droit du travail, Paris, Dalloz.

Bourdieu P. (1984), "La délégation et le fétichisme politique», Actes de la recherche en sciences sociales, $\mathrm{n}^{\circ} 52-53$, pp. 49-55.

CARTIER M. (2003), Les facteurs et leurs tournées : un service public au quotidien, Paris, La Découverte.

Castel R. (1995), Les Métamorphoses de la question sociale : une chronique du salariat, Paris, Fayard.

CÉlérier S. (2012), «Le salariat dans la chair. Ambivalences du tâcheronnat dans les industries de viande de volaille», in Cingolani P. (dir.), Un travail sans limites? Subordination, tensions, résistances, Toulouse, Érès, pp. 81-100.

Denis J.-M. (2008), «Conventions collectives : quelle protection pour les salariés précaires? Le cas de la branche du nettoyage industriel», Travail et emploi, $\mathrm{n}^{\circ} 116$, pp. 45-56.

DiDRY C. (2002), Naissance de la convention collective. Débats juridiques et luttes sociales en France au début du $20^{e}$ siècle, Paris, EHESS.

Duclos L., Mériaux O., (2009), «Métamorphoses de l'institution corporative et crise des politiques de l'intérêt», in Duclos L., Groux G., Mériaux O. (dir.), Les Nouvelles dimensions du politique. Relations professionnelles et régulations sociales, Paris, LGDJ, pp. 33-53.

Friot B. (2012), L'enjeu du salaire, Paris, La Dispute.

Grollier S. (2010), «Quelle syndicalisation des travailleurs de l'intérim?», Savoir/Agir, n 12, pp. 27-33.
Guillaume C., Pochic S. (2009), «La professionnalisation de l'activité syndicale : talon d'Achille de la politique de syndicalisation à la CFDT?», Politix, n 85 , pp. 31-56.

Habermas J. (1993), L'espace public : archéologie de la publicité comme dimension constitutive de la société bourgeoise, Paris, Payot.

Henry E. (2005), «Militer pour le statu quo. Le Comité permanent amiante ou l'imposition réussie d'un consensus », Politix, n 70 , pp. 29-50.

Higelé J.-P. (2012), «Les formes de la délibération interprofessionnelle : le sens du dialogue», Sociétés contemporaines, $\mathrm{n}^{\circ} 86, \mathrm{pp}$. 85-111.

Lagroye J., Offerlé M. (dir.) (2010), Sociologie de l'institution, Paris, Belin.

Lascoumes P., Serverin É. (1995), «Le droit comme activité sociale : pour une approche wébérienne des activités juridiques ", in LAscoumes P. (dir.), Actualité de Max Weber pour la sociologie du droit, Paris, LGDJ, pp. 155-177.

Michels R. (1971), Les partis politiques : essai sur les tendances oligarchiques des démocraties, Paris, Flammarion (1e éd. 1914).

Mischi J. (2011), "Gérer la distance à la "base". Les permanents CGT d'un atelier SNCF», Sociétés contemporaines, $\mathrm{n}^{\circ} 84$, pp. 53-77.

Offe C. (1985), Disorganized capitalism. Contemporary transformations of work and politics, Cambridge, The MIT Press.

Offerlé M. (1998), Sociologie des groupes d'intérêt, Paris, Montchrestien.

Pélisse J. (2003), «Consciences du temps et consciences du droit chez des salariés à 35 heures», Droit et société, $\mathrm{n}^{\circ} 53$, pp. 163-186.

PÉlisse J. (2009), «Judiciarisation ou juridicisation? Usages et réappropriations du droit dans les conflits du travail», Politix, n 86 , pp. 73-96.

Piotet F. (dir.) (2009), La CGT et la recomposition syndicale, Paris, Presses universitaires de France.

Rosanvallon P. (1998), La question syndicale, Paris, Hachette (1éd. 1988). 
Salais R. (2008), «Conventions de travail, mondes de production et institutions : un parcours de recherche», L'Homme et la société, $\mathrm{n}^{\circ}$ 170-171, pp. 151-174.

TAllard M. (2004), Action publique et régulation de branche de la relation salariale, Paris, L'Harmattan.

Thомаs A. (2011), «Universitaires engagés et nouveaux cadres syndicaux aux États-Unis : une alliance pour faire face au déclin des syndicats?», Genèses, $\mathrm{n}^{\circ} 84$, pp. 127-142.

Tournay V. (2011), Sociologie des institutions, Paris, Presses universitaires de France, coll. «Que sais-je?».

Vatin F., Bernard S. (dir.) (2007), Le salariat. Théorie, histoire et formes, Paris, La Dispute. 